Revue italienne d'études françaises

Littérature, langue, culture

$7 \mid 2017$

Figures littéraires de la haine

\title{
L'évidence du discutable. La rhétorique de la haine chez Corneilhan, Kimon et Loti
}

\section{Angela Di Benedetto}

\section{(2) OpenEdition}

\section{Journals}

\section{Édition électronique}

URL : http://journals.openedition.org/rief/1467

DOI : 10.4000/rief.1467

ISSN : 2240-7456

Éditeur

Seminario di filologia francese

Référence électronique

Angela Di Benedetto, «L'évidence du discutable. La rhétorique de la haine chez Corneilhan, Kimon et Loti », Revue italienne d'études françaises [En ligne], 7 | 2017, mis en ligne le 15 novembre 2017, consulté le 20 avril 2019. URL : http://journals.openedition.org/rief/1467 ; DOI : 10.4000/rief.1467

Ce document a été généré automatiquement le 20 avril 2019.

\section{(c) (i) (3)}

Les contenus de la RIEF sont mis à disposition selon les termes de la Licence Creative Commons Attribution - Pas d'Utilisation Commerciale - Pas de Modification 4.0 International. 


\title{
L'évidence du discutable. La rhétorique de la haine chez Corneilhan, Kimon et Loti
}

\author{
Angela Di Benedetto
}

1 Quand on considère sur une base collective les discours de la haine élaborés dans la France du XIXe siècle finissant, il n'est pas difficile de leur trouver un dénominateur commun et de les ramener à un modèle rhétorique bien précis, celui du pamphlet antisémite qu'avait inauguré Drumont (avec la France juive, 1886, et La libre Parole), et qu'avaient renforcé par la suite les écrits anti-dreyfusards. Ces libelles présentent effectivement une série de constantes idéologiques et morales consolidées avec le temps les Juifs sont accusés d'espionnage, de trahison, d'usure, de parasitisme ${ }^{1}$ - et visent l'efficacité par le biais d'une logique élémentaire sous-tendant l'argumentation (la logique de la conspiration, que Poliakov définit comme une causalité diabolique ${ }^{2}$ ), et également par la redondance de stéréotypes et d'images. Une grande partie de ces pamphlets martèlent sans arrêt des slogans du type «La France aux Français », «À mort le Juif ! », « Le Juif est un traître », « La Juive une putain », et se termine par une invective à laquelle est confiée la phase délibérative du discours (expulser les Juifs de la France). $\mathrm{Au}$-delà de tout jugement moral, on a là une littérature presque toujours pénible, et même fastidieuse. Presque toujours...

2 Car il arrive, en effet, que l'on tombe parfois sur des textes qui se distinguent des autres par la variété de leurs structures rhétoriques, leur taux figuratif ${ }^{3}$ et leurs intersections avec des registres et des codes littéraires de l'époque. Tel est le cas des trois pamphlets que j'entends traiter à présent, Juifs et opportunistes de Corneilhan ${ }^{4}$, Pathologie de l'Islam et les moyens de le détruire de Kimon ${ }^{5}$, Les Massacres d'Arménie de Loti ${ }^{6}$. Quoiqu'animés d'un sentiment d'hostilité à l'égard de la diversité de ces peuples - Juifs, Musulmans et Arméniens -, et avec des résultats littéraires différents, ces écrits combinent d'une manière plus intéressante les principes de la rhétorique ancienne et classique (logos, pathos et ethos). En particulier, ces trois pamphlétaires adoptent la leçon pascalienne selon laquelle pour que l'auditoire se rallie aux raisons de l'orateur, pour qu'il adhère 
" avec tout son cœur » à ses conclusions, « il faut que son cœur soit ému et qu'il s'identifie à la personne de l'orateur non moins que l'esprit épouse ses idées » ${ }^{7}$.

3 Par conséquent, identification et émotion. Identification avec un "nous » qui englobe, comme nous le verrons, celui qui écrit. Émotion conçue, en revanche, comme une finalité : susciter la haine, par une manipulation des sentiments et des idées de celui qui lit.

4 Comme il s'agit de discours délibératifs à raisonnements fallacieux ${ }^{8}$ visant à pousser l'auditoire à la haine, il n'est pas question seulement de convaincre (par la raison). Il faut persuader. Et la persuasion complète passe non pas par la preuve (preuve démonstrative) mais, comme Pascal lui-même le reconnaît, « par l'agrément " ${ }^{9}$.

5 Cette recherche d'agrément littéraire s'avère évidente quand on examine la structure qu'adopte le pamphlet Juifs et opportunistes. Corneilhan construit son discours sur le modèle d'une forme de narration qui s'affirmait alors en France au cours de ces mêmes années. Je veux parler du roman d'énigme ou policier, dont il adopte la méthode d'investigation, et plus précisément le paradigme indiciaire ${ }^{10}$.

$6 \mathrm{Au}$ début, il y a une constatation qui se donne comme étant incontestable dans son évidence : la France est en crise, proche de la catastrophe. La notion de crise génère une métaphore médicale, que l'on trouve souvent dans les pamphlets anti-juifs : le peuple français nous est montré comme un corps agonisant.

Il est un fait que nul ne songe à contester ; c'est l'état de crise générale dont souffre la France depuis dix ans et qui, s'aggravant sans cesse, semble toucher enfin à la période aiguë voisine des catastrophes. Ce peuple français si robuste et si expansif qui a pu cent fois renaître de ses cendres, toujours plus brillant et plus jeune, ce pays si vivace qui a résisté à tous les désastres, sent aujourd'hui dans son organisme des perturbations profondes. Comme un malade qui porterait dans ses flancs les germes mortels d'un mal inconnu, il souffre dans tous ses organes, recueille avec inquiétude les symptômes de son mal, et, ne pouvant trouver la cause pour la combattre, il finit par croire à sa décadence et désespérer de sa guérison. ${ }^{11}$

7 Le pamphlétaire observe la victime, l'anatomise, en lit les signes, invite à son tour le lecteur à en faire autant. Puis, le mystère tombe sur l'agent pathogène qui l'a réduite en cet état. Plus tard, sa nature envahissante et plurielle est évoquée par un "ils » générique : «ils sont les plus forts!» (mis sur les lèvres d'un hypothétique témoin de passage par la France). Mais le dévoilement est constamment différé par une argumentation qui, soustraite au rôle pour lequel elle a été fondée, à savoir parvenir à des conclusions par une démonstration, tend uniquement, au contraire, à identifier l'importance des maux subis par la victime et l'imminence d'une menace qui reste pourtant toujours indéterminée. On a affaire là à un raisonnement abductif conspirateur - c'est-à-dire à un raisonnement qui, à l'aide de simples indices, réduit les maux à une cause occulte unique - consacré presque exclusivement à l'argument de division ${ }^{12}$ : l'énumération de tous les corps sociaux infectés (la Magistrature, la politique étrangère, la politique intérieure, l'armée, les caisses de l'État, la Finance, la Justice, l'Industrie, l'Agriculture) démontrerait l'existence d'un organisme fortement dégradé, à l'intérieur duquel doit nécessairement se nicher un agent destructeur.

8 «Certes, pour qu'un pays tel que le nôtre soit si profondément atteint, pour que son économie tout entière soit si gravement troublée, il faut que notre mal soit bien redoutable $»^{13}$. 

qui argumente -, que l'énigme peut être résolue et la cause de la contagion dévoilée, de façon nette, lapidaire, comme une vérité incontestable, ignorée ou masquée depuis trop longtemps. Grâce à un saut logico-argumentatif violent, on abandonne la métaphore médicale pour se projeter dans la réalité quotidienne et fournir l'identité précise, concrète, de l'origine de ce mal obscur, et avec celle-ci, implicitement, une solution du problème. Il ne s'agit pas d'un adversaire indéterminé, quoique dangereux, mais d'un ennemi en chair et os, qui pourtant, en tant que tel, peut être attaqué et détruit: «Disons-le donc bien, ce fléau mystérieux qui nous mine, c'est le Juif, rien que le Juif ! » ${ }^{14}$.

Nous voici donc arrivés au faîte de ce climax savamment construit par l'écrivain pour stimuler la participation émotive du lecteur. L'effet qui en découle est double. On avait demandé au lecteur un investissement de l'intellect. Il peut à présent se détendre et jouir du plaisir que lui procure la résolution de l'énigme. Un plaisir intellectuel, renforcé dans ce cas par l'économie que lui assure une logique élémentaire et simplifiée, telle celle de la causalité diabolique ${ }^{15}$. De même que l'investigateur ramène tous les indices à l'action d'un seul criminel, de même Corneilhan ramène tous les maux du peuple français à un seul et unique coupable.

Mais le discours génère surtout un plaisir psychologico-émotif qui dérive du fait que cesse l'état anxiogène créé par une rhétorique de la terreur que l'on a su tourner vers la "sensibilisation » des consciences à l'aide de métaphores qui, parce qu'elles divisent le monde en victimes et bourreaux, proie et prédateurs, marionnettes et marionnettistes, fraudés et fraudeurs, Français et Juifs, polarisent et attisent les passions-émotions. Compte tenu de la loi du tiers exclu, si l'on n'est pas bourreau, si l'on n'est pas Juif, alors on est victime. L'identification avec la victime est ainsi inévitable pour le peuple français, lequel a été comparé en premier lieu à un malade qui se désespère de sa guérison parce qu'il ignore la cause de son mal, puis, à travers un raisonnement prévisionnel, à un « cadavre pantelant » jeté par Rotschild « aux pieds de l'Allemagne qui trouverait encore le moyen d'accommoder ses restes $»^{16}$. Cela a créé chez le lecteur un premier mouvement émotif, à savoir la sensation angoissante d'une catastrophe imminente. Mais la stratégie mise en œuvre par Corneilhan prévoit un second mouvement, celui du soulagementdétente apporté par la solution du problème. Une solution qui prévoit une action curative, directe, radicale : « divulguer par tous les moyens, [de] faire connaître à tous les Français la cause unique et secrète, l'infime et redoutable cause de nos malheurs; dès qu'elle sera connue, elle cessera d'être à craindre $»^{17}$.

13 C'est ici que se greffe le moment délibératif du discours. Au lecteur, armé de " courage », de la «conscience » d'avoir face à lui un ennemi et de celle de sa propre force, il est demandé de se ranger du côté du peuple français contre l'union israélite. Ce qui le persuade, ce n'est pas seulement l'appel aux valeurs patriotiques et civiles, mais aussi le recours à la polarisation métaphorique lumière/ténèbres, faiblesse/virilité qui lui impose un choix moral. 
même en se signant, n'osaient prononcer le nom du diable de peur d'évoquer son apparition $»^{18}$; ou bien, il peut s'identifier avec les voyants, les Wolski, les Drumont, les Meynier ${ }^{19}$. Il peut se reconnaitre en ces hommes qui, comme Corneilhan lui-même, ont décidé de mener une bataille au nom de la vérité et contre toute forme de mensonge. Très certainement, il faut agir, mais pour agir il est nécessaire de décider l'image de soi que l'on veut construire. L'enrôlement dans la phalange des "voyants ", des patriotes et des " hommes virils et robustes ", est le point d'arrivée du processus de reconnaissance de sa propre appartenance, un processus identitaire qui génère un inestimable plaisir moral et narcissique.

Dans Pathologie de l'Islam, pamphlet anti-islamique de Kimon, nous retrouvons le même mécanisme de persuasion pour le combat fondé sur le double mouvement de tension et distension émotives, quoique dans les limites d'une élaboration moins sophistiquée.

Tout le discours s'articule à partir d'une diabolisation de l'ennemi en vue de générer dans l'esprit du lecteur un état anxiogène indispensable à la prise de conscience de sa dangerosité. Pour en arriver là, Kimon s'en rapporte à la rhétorique traditionnelle qui accentue, en l'exaspérant, les traits menaçants du sujet à attaquer. Le peuple islamique est assimilé au règne animal, avec lequel il partage les comportements instinctuels, l'automatisme cérébral et la férocité2 ${ }^{2}$. C'est un peuple incivil qui a fermé ses portes au progrès, qui n'a jamais développé une tradition culturelle qui lui soit propre (contrairement à l'Occident, héritier de l'antique philosophie hellénique, chrétien, et dont la civilisation est fondée sur le travail et la productivité). Mais surtout, l'Islam est un sujet malade dont l'insanité réside dans une forme de folie anomale, « inclassable dans le groupe d'un ordre de folies connues par les aliénistes de l'Europe $»^{21}$.

17 Ce qui crée le climax de la tension émotive c'est moins le mystère sur l'identité de l'ennemi, dévoilée à l'instant que la nature hybride et mystérieuse de la maladie. Il s'agit, en effet, d'une folie "raisonnable » (la définition fait allusion à son ambivalence) causée par une modification pathologique du cerveau; une forme de dégénération supérieure qui comporte une perte du sens moral et qui pousse le sujet qui en souffre à une "systématisation d'idées de cruauté "2 $^{2}$. L'analogie avec les théories que les différentes branches de la médecine légale - la criminologie principalement - étaient en train d'élaborer en cette fin du XIX ${ }^{\mathrm{e}}$ siècle à propos d'une catégorie particulière de malade, un "type psychologique», l'individu cruel, mi-pervers, mi-pathologique, ne pourra échapper à quiconque fréquente la littérature médicale de l'époque ${ }^{23}$. Un individu qui préoccupait la communauté scientifique à cause de l'impossibilité de le cataloguer et, par conséquent, de le contrôler.

18 La tâche de susciter les émotions est à nouveau confiée au cliché que représente la maladie. Dans ce cas, comme la maladie coïncide avec l'élément pathogène, s'y ajoute un sentiment de répulsion. Mais, par rapport à Corneilhan où la « peur » de la maladie était excitée par un raisonnement analogique (la comparaison "comme un malade»), chez Kimon, la métaphore médicale cède la place à l'argumentation scientifique; ce qui comporte des implications significatives au niveau de l'argumentation. En effet, le cliché sort $\mathrm{du}$ domaine $\mathrm{du}$ pathos (auquel les pamphlets anti-juifs le reléguaient traditionnellement) et s'étend à l'ethos. Un ethos discursif dont le prestige, fruit de l'ostentation d'une étiologie en mesure de défier celle de Magnan, Charcot et KrafftEbing, sert d'argument d'autorité en faveur de la thèse proposée. Une étiologie hyperbolique, donc, dont les points essentiels sont les suivants : obsession du mot (« un soufflet mécanique dans le cerveau qui crache par la bouche le mot Allah! Ilah! ${ }^{24}$ ), 
manies systématisées ( «la circoncision, le jeûne du ramazan, la répulsion de la viande de porc, celle du vin, des cloches, de la musique, les ablutions »25), lypémanie («l'Islamisme rend l'individu mélancolique, ombrageux, morne. On peut dire que le Koran extirpe de l'homme jusqu'à la dernière racine de la bonne humeur, du sentiment de la joie ${ }^{26}$ ), perversion sexuelle ( l'instinct sexuel des Musulmans en général, est complètement dévié de la ligne normale : la perversion chez eux est purement psycho-pathique $\aleph^{27}$ ), hallucinations auditives ("à raison de la répétition continuelle d'une même idée délirante, l'idée koranique, le sens de l'ouïe joue un rôle prépondérant dans le cerveau mahométan ${ }^{28}$ ).

19 C'est le pathologiste, l'aliéniste, le psychologue criminel qui suggèrent le remède thérapeutique : l'Islam étant l'effet de la contagion d'une seule intelligence excentrique, d'un seul système cérébral, celui de Mahomet, il suffira de supprimer le culte de Mahomet pour mettre fin à la contagion. La proposition de transporter les cendres du prophète dans une urne que l'on aurait exposée au Louvre aurait représenté la victoire finale indiscutable - non dépourvue d'ironie - de l'Occident chrétien à l'égard d'un Orient dépossédé de son élément religieux et condamné à devenir une attraction exotique pour les visiteurs curieux du Louvre ${ }^{29}$.

Bien plus complexe est la stratégie de Loti, à tel point que l'on peut mieux la comprendre quand on adopte d'autres schémas herméneutiques, et tout particulièrement les principes interprétatifs de la nouvelle rhétorique élaborée par Perelman dans son Traité de l'argumentation, à partir précisément de l'exigence d'expliquer les mécanismes des raisonnements fondés sur des preuves non démonstratives attenant au champ du préférable ${ }^{30}$. Les Massacres d'Arménie est un opuscule anti-arménien inséré dans un pamphlet publié en 1919, Les Alliés qu'il nous faudrait. Dans ce libelle, Loti entendait soutenir l'alliance entre la France, qui aspirait à reconquérir son influence dans la Méditerranée, et la Turquie, menacée par la Russie. Par conséquent, la haine contre les Arméniens s'insère à l'intérieur d'une campagne organisée en vue d'accroître la capacité d'empathie envers les Turcs.

Différemment de Corneilhan et de Kimon, dont le schéma poussait presque mécaniquement le lecteur à haïr l'autre (avec des stratégies que nous avons illustrées plus haut), le mécanisme d'instigation à la haine élaboré par Loti est plus complexe. Il doit tenir compte que les deux sujets en jeu - les Turcs et les Arméniens, ennemis entre eux ne sont pas français comme le sont ses lecteurs et lui-même. Héritiers l'un et l'autre du grand empire ottoman, ils sont, aux yeux du lecteur, des étrangers, des corps étrangers. Pour susciter des émotions, Loti ne peut donc pas miser sur le sens de l'appartenance territoriale, sur la solidarité envers sa propre Nation. Et moins encore sur le facteur religieux. Les Arméniens sont des chrétiens, même s'ils ne sont pas d'observance catholique, alors que les Turcs sont musulmans. En outre, les Turcs viennent de se rendre coupables d'un second massacre envers les Arméniens, dix-neuf ans après celui qu'ils avaient déjà perpétré en 1894. Selon la logique binaire élémentaire, propre à toutes les rhétoriques du ressentiment, qui attribue le rôle tant de victime que de bourreau, les Turcs ne peuvent pas passer pour des victimes et susciter la compassion. Pour générer un sentiment d'hostilité envers les Arméniens et de sympathie pour les Turcs, l'auteur est dans l'impossibilité de proposer à son lecteur de s'identifier avec le peuple turc, bourreau et musulman, et de diaboliser le peuple arménien, victime et chrétien.

Le raccourcissement de la distance qui sépare les Français des Turcs ne peut s'obtenir qu'à la faveur d'un renversement du rapport victime-bourreau. Un renversement qui 
demande une opération, bien que moralement discutable, efficace cependant au niveau de l'argumentation. Loti traîne devant un hypothétique tribunal les deux peuples et les juge non pas sur la base des faits récents (dans ce discours judiciaire, il est significatif que le moment de la narration soit aboli car, comme il susciterait horreur et pitié, il serait de peu d'utilité pour la cause que l'on est en train de pérorer) mais sur l'image des deux peuples qu'il construit lui-même.

Le premier passage fondamental doit nécessairement prévoir le rachat de la mauvaise réputation des « amis » Turcs. Pour obtenir ce résultat Loti met en œuvre deux stratégies bien précises : la première, destruens, consiste à atténuer la gravité des crimes commis : l'importance du massacre est réduite; son atrocité est démentie et la responsabilité majeure est adossée à des tiers : les Kurdes, artisans matériels des pires crimes, et aussi les États européens, moralement responsables. Enfin, le caractère exceptionnel de l'événement est contesté et atténué sur la base de la motivation habituelle selon laquelle l'histoire humaine est constellée de massacres, et que les massacres des Européens dictés par le fanatisme religieux ont été pires. Loti recourt à la translatio criminis ${ }^{31}$ en présentant l'épuration ethnique non pas comme une attaque délibérée, mais comme la juste et inévitable réponse nationaliste à la menace que le peuple arménien représente pour l'Islam. Un peuple, l'Arménien - et cela constitue le dernier élément d'une construction rhétorique dont l'objectif est l'avilissement et l'outrage de ce peuple - n'ayant aucun nerf moral, rusé, double, ambigu, trompeur. Les Arméniens sont des traitres, des parasites, des profiteurs qui s'approprient des richesses de la nation turque. Ce sont des vers. Leur nature les assimile à une autre race maudite : «J'oserais presque dire que les Arméniens sont en Turquie comme des vers rongeurs dans un fruit, drainant à eux tout l'or, par n'importe quel moyen, par l'usure surtout, comme naguère les Juifs en Russie $»^{32}$. C'est la partie la plus scabreuse du pamphlet qui fut aussitôt censurée, ce qui est bien compréhensible.

La seconde stratégie qu'emploie Loti me semble plus intéressante. Une stratégie " constructive ", finalisée, dirais-je, à la création d'une relation d'empathie à l'égard du peuple turc au détriment du peuple arménien. Un résultat que Loti cherche à atteindre à l'aide d'un processus que je définirais d'hyperbole archétypale. J'entends, par là, l'exaltation des qualités innées de la civilisation ottomane dont le dénominateur commun est constitué par la valorisation de l'archaïque, du primitif, du rare (« un peuple qui retarde de quelques siècles sur le nôtre, - et je ne leur en fais point de reproches, bien au contraire $\left.»^{33}\right)$. La fascination du temps et de l'exotique, associés encore une fois à un Orient littéraire et esthétisé, un Orient abstrait et atemporel dépositaire de vertus antiques et précieuses à peu près inexistantes dans les sociétés modernes: loyauté, honnêteté, vénération de la part des enfants pour leurs parents, hospitalité et respect chevaleresque pour les hôtes, élégance morale, sensibilité envers tout le monde. Vertus qui jurent avec « notre Occident de doute et de cynisme, de tapage et de ferraille ».

Et c'est précisément sur le partage de ces valeurs, qui sont par ailleurs celles de la France monarchique et conservatrice, les seules auxquelles on puisse se référer sans déranger les consciences chrétiennes, que Loti fonde le rapprochement entre Turcs et Français. L'opposition pertinente, qui finit pour abolir toutes les autres, les rendant si non dérisoires du moins négligeables, est celle entre fidélité à la tradition et la trahison de ses valeurs. L'appel pour la Turquie devient celui à la Vieille France.

L'exorde du pamphlet affiche l'image de l'auteur comme celle d'un vieux sage, martyr, prophète, vox clamans in deserto, paladin titanique de la vérité, modèle de conduite à 
suivre $^{34}$ : un homme exemplaire et doté de virtus, qui a dû renoncer à sa compassion pour les Arméniens et devenir dur à leur égard parce que les faits lui ont été dévoilés, parce qu'il a découvert la vérité, une vérité que son éthique lui impose de faire connaître et dont il se fait explicitement le garant. Le lecteur instruit par lui et sans préjudices ne pourra que suivre le même parcours. La valeur de l'auteur du libelle constitue donc la prémisse «inexprimée » sur laquelle se fonde la prétention dont il fait preuve de se proposer comme modèle. Paraphrasant Perelman, nous pourrions dire que Loti impose « un comportement particulier» (le sien) non seulement pour «fonder ou [...] illustrer une règle générale », mais aussi pour « inciter à une action qui s'inspire de lui »35.

$\mathrm{Au}$ plaisir des sens suscité par l'évocation exotique de parfums, sons et paysages d'un Éden préservé par les Turcs (un univers caractérisé par des images de liquidité qui donnent une connotation de pureté : "l'eau qui court ", "on se sent comme baigné de paix et de confiance $»^{36}$ ) s'ajoute celui que procure ce mécanisme d'identification du lecteur moyen-médiocre avec l'auteur illustre du pamphlet, le moi qui discute et raisonne ${ }^{37}$. C'est le prestige de ce moi qui fonde la séduction d'un Nous qui unit Turcs et Français. Ce n'est pas un hasard si l'exorde et l'épilogue du pamphlet sont entièrement construits sur l'ethos (la plus puissante des trois "preuves " selon Aristote), un ethos qui faisant appel à l'argument d'autorité, détermine le ton et la cadence de son discours ${ }^{38}$. En se donnant comme modèle, Loti prescrit comme nécessaire le choix de son propre champ du préférable.

Tandis que Corneilhan et Kimon exploitent dans leurs argumentations des modalités tirées d'après la culture littéraire et scientifique contemporaine (l'enquête policière et la criminologie), Loti a recourt à une stratégie plus ancienne, celle fondée sur le principe d'autorité, assaisonnée d'un goût exotique fin de siècle. La différence réside dans le rôle que doivent jouer respectivement les lecteurs. Dans le cas de Loti le lecteur auquel il impose de "venir en nous " n'est pas impliqué directement dans l'énoncé contre un " eux », les ennemis mortels, mais seulement comme destinateur de l'acte d'énonciation. Il n'est pas la victime qu'on avertit de son propre danger mais il doit être le juge qui partage la vie d'un homme illustre qui affirme connaître les faits.

\section{NOTES}

1. Sur ce sujet il y a une large bibliographie. Cf., en particulier, les études de M. Angenot: M. Angenot, Un juif trahira : le thème de l'espionnage militaire dans la propagande antisémitique, 1886-1894, Montréal, Université McGill, 2003 ; Id., Ce que l'on dit des juifs en 1889 : antisémitisme et discours social , Montréal, C.I.E.E., 1984 ; Id., 1889 : un état du discours social, Longueil, Le Préambule, 1989.

2. L. Poliakov, Histoire de l'antisémitisme. L'Europe suicidaire : 1870-1933, Paris, Calmann-Lévy, 1977. Cf. aussi: P.-A. Taguieff, La Foire aux «Illuminés ». Ésotérisme, théorie du complot, extrémisme, Paris, Mille et une nuits, 2005; M. Angenot, Dialogues de sourds. Traité de rhétorique antilogique, Paris, Mille et une nuits, 2008 ; C. Passard, "Pensée du complot et imaginaire judéophobe chez E. Drumont », dans E. Danblon-L. Nicolas (dir.), Les rhétoriques de la conspiration, Paris, CNRS, 2010, p. 195-216. 
3. Pour la notion de taux figuratif cf. F. Orlando, Per una teoria freudiana della letteratura, Torino, Einaudi, 1987.

4. G. Corneilhan, Juifs et opportunistes. Le judaïsme en Égypte et en Syrie, Paris, Sauvaître, 1889.

5. D. Kimon, La pathologie de l'Islam et les moyens de le détruire: étude psychologique, Paris, Kimon, 1897.

6. P. Loti, «Les massacres d'Arménie », dans Id., Les alliés qu'il nous faudrait, Bayonne, Foltzer, 1919.

7. M. Angenot, Dialogues de sourds. Traité de rhétorique antilogique, cit., p. 61.

8. Dans la plupart des cas, il s'agit de raisonnements abductifs, fondés sur des prémisses discutables (non pas vérité mais évidence), et d'inférences non valables, où l'abduction - comme l'a bien montré Danblon à propos de la rhétorique anti-juive - « livrerait un passage immédiat à une conclusion sans devoir en passer par l'examen des prémisses» (E. Danblon, «Les "théories du complot" ou la mauvaise conscience de la pensée moderne », dans E. Danblon-L. Nicolas (dir.), op.cit., p. 57-72, p. 60). Sur la notion d'évidence, cf. M. Angenot, Dialogues de sourds. Traité de rhétorique antilogique, cit., p. 182.

9. «De sorte que l'art de persuader consiste autant en celui d'agréer qu'en celui de convaincre, tant les hommes se gouvernent plus par le caprice que par raison!» (B. Pascal, De l'art de persuader, Paris, Mille et une nuits, 2001, p. 6).

10. Cf. C. Ginzburg, «Spie. Radici di un paradigma indiziario », dans Miti, emblemi, spie. Morfologia e storia, Torino, Einaudi, 1986.

11. G. Corneilhan, op. cit., p. 7-8.

12. Cf. Ch. Perelman, L. Olbrechts-Tyteca, Traité de l'argumentation. La nouvelle rhétorique, Bruxelles, Éd. de l'Université de Bruxelles, 1976, ch. 56, p. 316-317.

13. G. Corneilhan, op. cit., p. 11.

14. Ibid., p. 13.

15. M. Angenot, Dialogues de sourds. Traité de rhétorique antilogique, cit., p. 339. Sur ce point, cf. aussi : Id., «La pensée conspiratoire. Une histoire dialectique et rhétorique ? ", dans E. DanblonL. Nicolas (dir.), op. cit., p. 25-42; P.- A. Taguieff, op. cit.

16. G. Corneilhan, op. cit., p. 17.

17. Ibid., p. 12.

18. Ibidem.

19. Ibid., p. 19-20.

20. Dans un autre de ses pamphlets Kimon attribue les mêmes symptômes aux juifs. Cf. D. Kimon, L'influence juive produit l'Automatisme cérébral, Valence, 1898.

21. Id., La pathologie de l'Islam et les moyens de le détruire : étude psychologique, cit., p. 51.

22. Ibid., p. 59.

23. Cf. A. Di Benedetto, «Il paradosso della crudeltà a fine Ottocento tra scienza e letteratura ", dans Rivista di Letterature Moderne e Comparate, LIX, 2006, p. 435-447.

24. D. Kimon, La pathologie de l'Islam et les moyens de le détruire :étude psychologique, cit., p. 55.

25. Ibid., p. 57.

26. Ibid., p. 58.

27. Ibid., p. 60 .

28. Ibid., p. 60-61.

29. «Donc, pour détruire l'Islam, il faut supprimer le centre d'action de l'Islam, c'est-à-dire La Mecque et s'emparer, à Médine, des restes du Prophète Mahomet, les transporter au Musée du Louvre, et sur sa dépouille on inscrirait ces mots pour l'éternité : Ci-git l'Islam ! Né à La Mecque en 612 et supprimé en 1897 » (Ibid., p. 183).

30. Ch. Perelman, L. Olbrechts-Tyteca, op. cit., paragraphes18-24. 
31. M. T. Cicéron, « Rhétorique à Herennius ", dans CEuvres complètes, vol. 1, 1. I, XV, Paris, Garnier, 1866, p. 42.

32. P. Loti, op. cit., p. 47.

33. P. Loti, op. cit., p. 41. Cette stratégie n'est pas sans rapport avec celle de la « représentation de l'âge d'or » dévoilée par Perelman et Angenot. Cf. Ch. Perelman, L. Olbrechts-Tyteca, op. cit., ch. 57, p. 330-331; M. Angenot, La parole pamphlétaire. Typologie des discours modernes, Paris, Payot, 1982, p. 106-107.

34. Sur l'image de l'énonciateur cf. M. Angenot, La parole pamphlétaire. Typologie des discours modernes, cit., ch. 3.1-3.

35. Ch. Perelman, L. Olbrechts-Tyteca, op. cit., ch. 80, p. 488.

36. P. Loti, op. cit., p. 41 et p. 51.

37. Sur le mécanisme d'identification du lecteur avec l'écrivain dans ce pamphlet de Loti cf. aussi mon étude : "Giustificare l'ingiustificabile. Analisi argomentativa dei «Massacres d'Arménie" di Pierre Loti », dans S. Nienhaus-D. Mugnolo (dir.), Questione armena e cultura europea, Foggia, Grenzi, 2013, p. 173-201.

38. Pour le terme " ethos", nous désignons, avant tout, le signifié de «image que le locuteur construit de lui-même ». Mais, dans le cas de Loti, il est légitime aussi de parler d'ethos préalable (ou pré-discursif), car l'image élaborée de l'écrivain s'appuie soit sur des stéréotypes qu'il s'attribue à lui-même dans l'exorde (qui, selon la rhétorique ancienne et classique, précède l'argumentation), soit sur des éléments préexistants à sa prise de parole, comme l'autorité que lui confère son statut d'académicien français, ou sa réputation d'écrivain à succès. Sur les notions d'ethos, d'ethos préalable ou pré-discursif et d'image de soi, cf. R. Amossy (dir.), Images de soi dans le discours : la construction de l'ethos, Lausanne, Delachaux et Niestlé, 1999 ; Id., La présentation de soi : ethos et identité verbale, Paris, Presses universitaires de France, 2010 ; Id., L'argumentation dans le discours : discours politique, littérature d'idées, fiction, Paris, Nathan, 2000.

\section{RÉSUMÉS}

Cet article, centré sur l'analyse des trois pamphlets, Juifs et opportunistes de Corneilhan, La pathologie de l'Islam de Kimon, Les alliés qu'il nous faudrait de Loti - porte-parole du sentiment antijuif, anti-islamique et anti-arménien -, tend à dégager les caractères spécifiques de ces textes par rapport au modèle de discours de la haine qu'avait inauguré Drumont à la fin du XIX siècle, dont ils portent par ailleurs des traces évidentes. En effet, bien qu'ils tirent de nombreux topoï et stéréotypes de la rhétorique du complot qui s'était affermie à l'époque de l'Affaire Dreyfus, ce qui distingue les trois pamphlets de cette rhétorique-là, ce sont la variété des structures rhétoriques, le taux figuratif et les recoupements avec les registres et les codes littéraires de l'époque.

\section{INDEX}

Mots-clés : Loti (Pierre), Corneilhan (Georges), Kimon (Daniel), discours de la haine, argumentation, rhétorique 